\title{
CONSECUENCIAS DEL CONVENCIONALISMO EN LAS TEORÍAS GEOMÉTRICAS DEL ESPACIO-TIEMPO
}

\author{
Albar GaRCía DE GURTUBAI ESCUdERO \\ Telescopio Nazionale Galileo. (Santa Cruz de la Palma)
}

RESUMEN. El desarrollo de las geometrías no euclídeas a lo largo del siglo XIX contribuyó, en su día, a descartar cualquier pretensión de determinar la geometría del espacio-tiempo, cimentando, de este modo, las bases del convencionalismo de Poincaré. Sin embargo, el convencionalismo parece vacilar entre una versión fuerte que propone la equivalencia completa entre distintas teorías, y una versión débil que se limita a defender la equivalencia empírica. En el presente artículo trataré de dar a entender las consecuencias epistemológicas de ambas versiones del convencionalismo desde tres perspectivas diferentes: realista, positivista lógica y pragmática. Así mismo, se criticará la idea, según la cual, la posibilidad de que todos y cada uno de los teoremas y proposiciones de una teoría geométrica sean traducibles en términos de los teoremas y proposiciones de otra teoría es suficiente para inferir la equivalencia completa. Finalmente, se defenderá la versión débil del convencionalismo argumentando sobre la base de las teorías geométricas del espacio-tiempo que emanan de la teoría newtoniana y de las teorías relativistas.

ABSTRACT. The development, of the non-euclidean geometries throughout the century XIX, contributed to discard any pretension to determine the geometry of the space-time, establishing, thus, the bases of Poincare's conventionalism. Nevertheless, the conventionalism seems to hesitate between a strong version that proposes the complete equivalence between different theories, and a weak version that limits itself defending the empirical equivalence. In this paper I 
will try to explain the epistemological consequences on both versions of the conventionalism from three different perspectives: realistic, logical positivism and pragmatism. Likewise, I will criticize the idea, as which, each and every of the theorems and propositions of a geometrical theory are translatable in terms of the theorems and propositions of another theory it is sufficient to infer the complete equivalence. Finally, I will defend the weak version of the conventionalism arguing on the base of the geometrical theories of the space-time that come from the Newtonian theory and from the theories of relativity.

\section{Introducción}

Históricamente, la cuestión acerca del fundamento ideal para el conocimiento del mundo que se derivó de la revolución científica del siglo XVII siguió dos derroteros distintos. En primer lugar se pensó que toda proposición podía conocerse con un grado razonable de certeza siguiendo las directrices únicamente de la razón. En segundo lugar fue imponiéndose la idea de que el conocimiento del mundo, las verdades generales y teóricas acerca de la naturaleza de las cosas, podían sólo ser inferidas a partir de verdades fundamentales que descansaban en los datos suministrados a través de la observación y de la experiencia. Esta inferencia era fiable, pero falible.

En este orden de cosas la geometría seguía siendo una disciplina incómoda de cara a este enfoque empirista de la teoría del conocimiento. Si se supone que la geometría aporta verdades incuestionables sobre la naturaleza del mundo porque se derivan, a través de inferencias lógicas, de verdades fundamentales incuestionables y cognoscibles por la razón pura, entonces se podría pensar que la geometría ofrece conocimiento verdadero con independencia de la observación y de la experiencia. Cabe ahora preguntarse si no sería posible que en algún momento todo nuestro conocimiento físico llegara a descansar en dicho fundamento epistemológico superior.

El desarrollo de las geometrías no-euclídeas, en el siglo XIX contribuyó a desechar las pretensiones de este tipo. Visto que la geometría euclídea no era la única geometría posible, ¿̇e qué modo se podía afirmar que las verdades de la geometría podían desarrollarse con independencia de la experiencia? En este caso, 
¿no se debería hacer uso de la observación, como habitualmente se hace en otras ciencias, para averiguar cuál de las posibles geometrías describe verdaderamente la estructura del mundo?

Los defensores de la idea según la cual la geometría euclídea es la descriptora del mundo cuestionaron la propia consistencia lógica de las geometrías noeuclídeas. Esta tentativa fracasó de inmediato, dado que no se tardó en crear pruebas de consistencia para las geometrías axiomáticas no-euclídeas. Según dichas pruebas se podía estar seguro por pura lógica de que si las geometrías no-euclídeas eran inconsistentes, también debía serlo la geometría euclídea. Aún con todo lo dicho, los kantianos pudieron seguir defendiendo, por otras razones, que la geometría euclídea era la verdadera descriptora del mundo, manteniendo la idea de que la verdad de la geometría euclídea conllevaba un tipo de necesidad que transcendía a la necesidad de las verdades cuya certeza se debía únicamente a la lógica. En general, sin embargo, quienes estaban familiarizados con la existencia de las nuevas geometrías, estaban convencidos de que la geometría del mundo, como su química o su física, era algo sobre lo que sólo la experimentación nos podía informar.

Con el advenimiento de la relatividad las geometrías no-euclídeas comenzaron a jugar, de hecho, un papel importante en la física teórica. El sendero comienza con el espacio y el tiempo newtoniano, continúa a través del espacio-tiempo de la relatividad restringida, y nos lleva hasta el espacio-tiempo curvo de la relatividad general. En cada periodo de transición los hechos empíricos, los resultados de la observación y de la experiencia, jugaron un papel decisivo. Los postulados del espacio-tiempo de la relatividad restringida descansan sobre el descubrimiento de que la velocidad de la luz parece ser la misma en todos los estados inerciales de movimiento y en todas las direcciones, como se deduce de los experimentos de ida y vuelta en el interferómetro Michelson-Morley. Por otra parte, el hecho de que la velocidad de la luz fuese la velocidad máxima de propagación de las señales causales tuvo también un papel importante en la fundamentación de la teoría, siendo esto también un hecho de la experiencia observacional. Se pueden añadir todos los hechos predichos con relación a la teoría restringida, como los relativos a los tiempos de vida aparentes de las partículas inestables en movimiento respecto al observador, el aumento aparente de la masa inercial con el aumento de la velocidad, y otros que se tomaban como fundamentación experimental de una nueva imagen del espacio-tiempo. 
Así mismo, en el paso a la relatividad general los hechos derivados de la observación jugaron un papel fundamental, primero al sugerir la teoría, y después al confirmarla. Las experiencias realizadas por Galileo, sobre cómo la gravedad actuaba sobre los objetos independientemente de su tamaño y constitución, sugirieron a Einstein la idea de tratar la gravedad como una característica geométrica del espacio-tiempo. Las acertadas predicciones acerca de la desviación de las trayectorias de los rayos de luz en las inmediaciones de campos gravitatorios intensos y las velocidades relativas de los relojes ideales en lugares diferentes de un potencial gravitatorio se consideran como confirmación de la teoría. Otra confirmación observacional la dan los pequeños cambios predichos en las órbitas de los planetas respecto a las trayectorias inferidas por la teoría newtoniana. A la larga, y en función del desarrollo tecnológico se espera poder realizar observaciones a escala astronómica, e incluso a escala cosmológica, observando la estructura globalmente curvada de la geometría del universo, tratando de seguir probando las predicciones de la teoría, y en caso de concordancia confirmar su veracidad.

Hasta aquí parece evidente que los empiristas tenían razón. Pudiéndose imaginar un número ilimitado de geometrías para el universo sólo la observación nos podría dar información de cuál es la correcta. Esto parece tan cierto para la geometría como para la física, la química, o la psicología. Parece que cualquier esperanza de conocer la geometría del mundo con certeza e independientemente de la observación es una falacia.

Pero las cosas no están tan claras para todo el mundo. Por lo menos eso se desprende de los análisis realizados por Henri Poincaré.

\section{Convencionalismo}

Poincaré sugirió, en un excelente estudio sobre estatus del conocimiento geométrico, la posibilidad de que el conocimiento de la geometría del mundo no viniera determinado por la experiencia. Su análisis precedió en unos pocos años a la relatividad y arrojó mucha luz sobre el papel de la geometría en esta nueva teoría. Comenzó presentando en una serie de ensayos pruebas de consistencia relativa para la geometría no-euclídea, refutando así, cualquier afirmación al respecto de que dichas geometrías deban ser rechazadas por ser lógicamente inconsistentes. A continuación considera la idea kantiana de que la geometría euclídea es la necesariamente válida para el mundo. Realiza dicha reivindicación 
considerando que la necesidad de esta geometría se basa en el hecho de que el espacio es un componente de nuestra percepción del mundo y esta geometría describe la estructura de lo percibido que es dado a la percepción a través de la mente de quien percipiente. Poincaré arguye que el espacio de la física, aquel en el que se dan los acontecimientos materiales, debe distinguirse del espacio perceptual, tal como el llamado campo visual de la percepción visual. Afirma, igualmente, que no sabemos nada de la existencia o naturaleza del espacio físico por ninguna experiencia perceptual directa, sino más bien, por inferencia de aquello que percibimos directamente. Es el orden y la regularidad, afirma Poincaré, de nuestras experiencias perceptuales de los fenómenos, lo que nos conduce a suponer que estas experiencias tienen un origen causal en los acontecimientos físicos. Es decir, inducimos la existencia del mundo físico, incluyendo el espacio y su naturaleza como una hipótesis que da explicación del orden y regularidad que experimentamos en nuestras percepciones directas. Es una inferencia realizada a partir de una hipótesis.

Se podría esperar que Poincaré propusiera que la geometría se soporta en la inferencia razonable a partir de los datos de la observación y que se podría, de hecho, descubrir que las geometrías no-euclídeas se ajustan mejor a los datos observacionales que la euclídea. Pero en vez de esto, Poincaré nos sorprende con un argumento orientado a concluir que la geometría euclídea no puede ser refutada por ningún tipo de experiencia. Incluso afirma que siempre se considerará a la geometría euclídea como la geometría del mundo. Ésta tiene así, un tipo de necesidad, que es sólo cuestión de determinación convencional. No es tan solo el reflejo de un hecho metafísico acerca del mundo.

A fin de defender sus argumentos, Poincaré propone un mundo imaginario que hoy se conoce como disco de Poincaré. Imaginemos seres bidimensionales que habitan en un disco finito euclídeo. Estos seres tratan de determinar la geometría de su mundo llevando consigo reglas, escuadras y cartabones. Pero desde fuera les engañamos. Sus instrumentos se expanden y contraen en función de la temperatura - todos del mismo modo-y ajustamos la temperatura del disco de manera que los objetos se contraigan a longitud cero en el borde del disco. Con un decaimiento adecuado de la temperatura, desde el centro hasta el borde, es fácil inducirles a engaño haciéndoles creer que habitan en un universo bidimensional infinito lobachevskiano curvado negativamente. Si intentaran utilizar trayectorias de luz para averiguar la geometría de su universo, nos bastaría 
con colocar un medio con índice de refracción variable que curvara las trayectorias de los rayos de luz para mantenerlos en el mismo engaño. Así, estos extraños seres serían siempre burlados, desde fuera, concluyendo que la geometría del universo que habitan es no-euclídea, cuando realmente no es así.

Hagamos ahora la extrapolación a nuestro mundo tridimensional (o tetradimensional, si consideramos el espacio-tiempo). Con independencia de las mediciones que se hagan utilizando reglas y rayos de luz (o partículas libres y relojes, alternativamente), ¿qué garantías se pueden tener de que cualquier apariencia de no-euclicidad en la geometría sea real, y no una yuxtaposición de geometría euclídea y campos de dilatación y contracción?

En el caso de las criaturas bidimensionales, nosotros mismos tenemos la última palabra acerca de lo que realmente está ocurriendo en el universo del disco. En nuestro caso, pretendemos determinar la geometría de nuestro universo, y cabe preguntarse, ¿qué es lo que debe marcar la diferencia entre una geometría real no-euclídea y un mundo euclídeo con campos distorsionantes que afectan a todos los instrumentos, posibles, de medida? Poincaré nos sugiere que no hay ninguna posibilidad, dados los hechos implicado, que determine cuál de las hipótesis es la correcta. Nos corresponde a nosotros decidir qué descripción dar al mundo. La verdadera geometría del universo se convierte ahora en una cuestión de decisión o convención por nuestra parte. Poincaré llega a sugerir que dado que la geometría euclídea es la más sencilla, siempre la elegiremos como verdadera, restaurando así, la euclicidad como característica necesaria del mundo, o más bien, convencionalmente necesaria. Podría pensarse, por otra parte, que es más sencillo describir el mundo en términos no-euclídeos si tuviéramos a mano una teoría que simplificara el resto, como es el caso de la relatividad general con su espacio-tiempo curvo.

Aún con todo, el argumento de Poincaré es claro. Las evidencias acumulables mediante la observación requieren una explicación teórica. Pero las hipótesis que ofrecemos para explicar las observaciones tienen una estructura. Podemos dar una alternativa acerca de la constitución de una parte de la estructura, sean cuales sean las observaciones, siempre y cuando seamos lo suficientemente flexibles para acometer cambios en otra parte de la estructura. Por consiguiente, terminará siendo una cuestión, no de hecho, sino de convención el que una geometría, y no otra, describa el espacio-tiempo. 


\section{Respuestas al convencionalismo}

Se puede, no obstante, hacer una crítica a los planteamientos de Poincaré. Principalmente, se puede negar la premisa fundamental, según la cual, es aislable un conjunto de supuestos hechos sobre el mundo como hechos de observación pura y situar luego estos hechos en el dominio de las percepciones de la mente. Se podría negar que tal dominio de lo percibido directamente sea inteligible, diciendo que el conjunto de nuestras percepciones es del mundo físico, y no de algunos datos sensoriales en nuestras mentes. Se negaría también la existencia de objetos cognoscibles con independencia de una teoría postulada sobre el mundo. Al fin y al cabo, ¿podemos creer en serio que el espacio en el que vemos mesas y sillas no es el espacio físico, sino un espacio visual, táctil, o motriz que no pertenece al dominio de la física?

También se podría negar la afirmación de que hay hechos inmunes a ser comprobados por observación directa. Para Poincaré es crucial que hechos como que el espacio del mundo sea realmente plano o curvo puedan únicamente conocerse por medio de inferencias y no puedan ser determinados por ningún tipo de inspección directa. Esta inmunidad de los hechos geométricos a la observabilidad preserva la posibilidad de teorías alternativas que sí salvaguardan los fenómenos observables. Precisamente, esta inmunidad está en la raíz del argumento de Poincaré de que nunca podremos afirmar con legitimidad que conocemos la geometría del mundo.

La crítica a esta inmunidad por siempre a la inspección directa viene de un análisis de lo que antaño fueran inobservables y hoy no lo son. Acaso, ¿̨no podemos ver bacterias utilizando microscopios ópticos, virus con microscopios electrónicos, o átomos con microscopios de efecto túnel? ¿Cómo puede estar Poincaré tan seguro de que nunca podremos observar si nuestro espacio-tiempo es plano o curvo?

La suposición de que las estructuras geométricas forman parte de un reino permanentemente inmune a la observación directa puede tener un gran poder persuasivo si uno se plantea la pregunta del siguiente modo. ¿Que significado tiene observar, no ya rayos de luz, partículas, reglas o relojes, sino la estructura del espacio-tiempo mismo? Esta cuestión entraña una gran cantidad de enigmas. Acaso, ¿no observamos directamente intervalos temporales entre nuestras experiencias? 
Una vía de argumentación en favor de Poincaré es que casi toda la física contemporánea que se ocupa del espacio y del tiempo descansa en el mismo tipo de aserción referida a la inmunidad de algunos hechos a la observación directa. Cuando Einstein critica en la teoría de la relatividad restringida la noción de simultaneidad de sucesos distantes presupone que dicha simultaneidad ha de ser determinada por medio de la luz, por señales causales de algún tipo, o transportando relojes. No puede considerarse como algo abierto a la inspección directa. Incluso en los fundamentos de la relatividad general, a la hora de geometrizar la gravedad postula que el campo gravitatorio puede ser conocido tan solo por sus efectos. Lo que realmente se observa es el comportamiento de los rayos de luz, de las partículas, de las reglas y de los relojes, y no el campo gravitacional propiamente dicho. En este caso particular de la relatividad parece haber dos suposiciones infiltradas en sus fundamentos. Primero, lo que observamos es el comportamiento de cosas materiales, no la estructura del espacio-tiempo mismo. Segundo, únicamente podemos determinar observacionalmente el comportamiento de objetos materiales en un momento dado, es decir, cosas tales como que los extremos de dos reglas rígidas coinciden uno con otro. No se puede considerar como un hecho observacional el que dos reglas rígidas separadas una cierta distancia tengan, o no, la misma longitud. Del mismo modo, no se puede considerar la simultaneidad a distancia como una característica observable del mundo en el sentido de la observabilidad directa.

Podría darse el caso de que estas suposiciones acerca de características del mundo inmunes por siempre a la determinación observacional directa estén equivocadas. Pero en cualquier caso, se presuponen en el análisis que fundamenta nuestra aceptación de las teorías del espacio-tiempo contemporáneas. Supongamos, pues, que Poincaré está en lo cierto respecto a estas estructuras inobservables.

El hecho de que nuestras estructuras postuladas trasciendan a los hechos observables introduce en las teorías una ambigüedad que permite a numerosas versiones alternativas tener las mismas consecuencias observacionales. ¿Por qué motivo, entonces, se da por válida la relatividad frente a otras teorías? En la teoría restringida, mientras la coincidencia de sucesos es observable, la simultaneidad de sucesos distantes se obtiene postulando la constancia de la velocidad de la luz con independencia del sistema inercial y en todas las direcciones, hecho éste, que no puede comprobarse observacionalmente. Otra vez, para tener la estructura espacio-temporal completa de la relatividad restringida hay que aña- 
dir el postulado de linealidad, o lo que es lo mismo, postular que el espacio-tiempo es plano. Sólo formulando estos postulados obtenemos la teoría estándar y no la vieja teoría de un éter absoluto con reglas y relojes compensatorios que se contraen y atrasan realmente cuando están en movimiento. Por su parte, en la teoría general se postula que los rayos de luz curvados y las partículas libres describen bajo el influjo de la gravedad, geodésicas nulas y cronomorfas del espacio-tiempo respectivamente, considerando las reglas y relojes locales como correctos indicadores de los intervalos métricos del espacio y tiempo. Sólo bajo estas hipótesis se consigue que las observaciones de los efectos de la gravedad hagan inferir que el espacio-tiempo tiene curvatura, cuadrando la teoría con las observaciones de objetos materiales. Con otros postulados se podría preservar la estructura espacio-temporal plana de la relatividad general, considerando que la gravedad es un campo de fuerzas yuxtapuesto que tiene efectos métricos y dinámicos sobre las partículas en movimiento y sobre la luz.

La idea de Poincaré describe muy bien el contexto en que fueron desarrolladas las teorías relativistas. Se obtienen nuevos hechos observacionales y estos están restringidos, local y materialmente, sobre nuestros instrumentos de medida. Para justificar todo esto se postula una estructura espacio-temporal, pero éstas sobrepasan con su riqueza la totalidad de los hechos observacionales que las soportan. Igual que en el disco de Poincaré tenemos ahora una variedad de binomios teoría-estructura geométrica.

\section{Consideraciones realistas}

Si nos negáramos a descartar el problema de Poincaré y nos lo tomáramos en serio, ¿cómo deberíamos continuar? Una posibilidad es aceptar abiertamente que las teorías del espacio y del tiempo proponen verdaderas estructuras reales, pero inobservables, del mundo. En este sentido, estaríamos asumiendo que sólo una de las múltiples teorias posibles del espacio-tiempo compatibles con los datos observados es la correcta. Argüiríamos que tan sólo los hechos observacionales pueden servirnos de guía legítima a la hora de elegir una teoría como válida frente a las demás. Finalmente, argüiŕamos que simplemente debemos negarnos a emitir un juicio sobre cuál de las teorías espacio-temporales alternativas describe realmente el mundo. Estaríamos así, haciendo frente honestamente a nuestras limitaciones y rechazando un supuesto conocimiento de lo que simplemente no podemos conocer. 
Para eludir este camino al abandono podríamos buscar apoyo en otras características de las teorías que usamos a fin de seleccionar la mejor y más convincente dentro del conjunto de alternativas entre las que se puede hacer elección por compatibilidad con los datos observados. Al realizar dicha elección de una teoría se aplican, generalmente, consideraciones que sobrepasan la mera comparación de las consecuencias observacionales de la teoría con los hechos observados. Éstas son consideraciones que no sé hasta que punto pueden ayudarnos a decidir con cierta legitimidad cuál es la teoría más acertada.

Suele defenderse la idea de que unas teorías tienen una mayor plausibilidad intrínseca que otras. Otra idea que suele servir de guía en la elección de teorías es la relación que presenta con la ciencia de fondo sobre la que se proyecta y que se supone que puede servir para discernir entre teorías más fiables y aquellas en las que no merece la pena confiar.

A este segundo tipo de enfoque pertenece la consideración de que el conservadurismo metodológico puede ser una guía para dar preferencia a una teoría sobre otras observacionalmente indistinguibles de ella. Se sostiene aquí que existe una regla del método científico en el sentido de que al elegir una nueva teoría lo hacemos considerando cuál se desvía menos de las viejas teorías que venían siendo aceptadas hasta el momento, y que ahora rechazamos debido a su incompatibilidad con nuevas evidencias observacionales.

Apliquemos esta regla al caso de la relatividad general. Estamos buscando una nueva teoría de la gravedad. La teoría de Newton es incompatible con la gravedad y debe ser rechazada, pero la teoría nueva que venga a substituir a la antigua ha de ser compatible con los nuevos datos observacionales y que se acerque en sus afirmaciones, en la medida de lo posible, a lo que la teoría vieja afirmaba. ¿Nos lleva dicha regla metodológica a elegir la teoría de Einstein frente a las teorías alternativas de dilatación y contracción más campos de fuerzas? Sin lugar a dudas es cierto que en la vieja teoría usábamos reglas y relojes para indicar correctamente intervalos espaciales y temporales. Esto sigue siendo cierto en la relatividad general, pero no en su alternativa espacio-temporal plana. Parece que el conservadurismo metodológico nos lleva a seleccionar la relatividad general como alternativa razonable.

Sin embargo, en la vieja teoría el espacio-tiempo era plano. Esto sigue siendo válido en la alternativa del campo gravitatorio, pero en la relatividad general 
se propone un cambio radical dando curvatura al espacio-tiempo. Desde este punto de vista parece que la alternativa de espacio-tiempo plano más campo gravitatorio es la elección más conservadora y por consiguiente, la que ha de preferirse. El problema estriba, claro está, en que la idea de ser conservador respecto a la vieja teorfa es ambigua. Hay muchos aspectos en las viejas y nuevas teorías. El ser conservador respecto a algunos de estos aspectos nos conduce a elegir una de las alternativas, y el ser conservador respecto a otros aspectos nos lleva a una elección diferente. Se convierte así, la regla del conservadurismo en un criterio de elección arbitrario. ¿Puede ser este criterio, acaso, una guía fiable a la verdad?

No obstante, existen más criterios de elección. Algunos nos permiten apoyarnos en características intrínsecas de las teorías. Uno de ellos considera la noción de simplicidad teórica. Se afirma habitualmente que los científicos, dadas dos alternativas teóricas entre las que los datos observacionales no son decisivos, elegirán la más simple de las dos, revelando una intuición de que la hipótesis más simple es más probable que sea la hipótesis verdadera.

La noción de simplicidad es, no obstante, terriblemente complicada. En algunas versiones depende de la manera en que se formule la teoría. Según la forma de ser representada, una teoría podría parecer más simple que otra, pero dicho orden de simplicidad se podría ver trastocado si las teorías se expresaran de otra manera. Otras nociones de simplicidad, atienden a las características estructurales de la teoría. Siendo la forma lógica de sus premisas básicas un atenuante de la aparente relatividad de la simplicidad respecto a su forma de expresión. Según esta noción de simplicidad la teoría de la relatividad restringida es más simple que las alternativas de éter. Así mismo, la relatividad general gana en simplicidad, bajo estos criterios frente a las teorías de espacio-tiempo plano más campos. En estos dos casos las teorías de Einstein no contienen la estructura problemática e innecesaria que contamina a sus alternativas.

Para ver esto, es preciso comparar, primero, el espacio-tiempo que emana de la relatividad restringida con una de las teorías del éter. Einstein postula que la velocidad de la luz en la misma en todas direcciones y en todos los sistemas de referencia inerciales. La teoría del éter lo niega defendiendo que la luz tiene una velocidad constante en todas direcciones únicamente en el sistema que permanece en reposo respecto al éter. En todos lo demás sistemas de referencia la luz parece tener la misma velocidad en cada dirección sólo debido al efecto del movi- 
miento de laboratorio con respecto al éter sobre instrumentos de medida tales como reglas y relojes. La diferencia, es que mientras Einstein atribuye los resultados nulos de los experimentos de ida y vuelta en el interferómetro MichelsonMorley a la constancia de la velocidad de la luz en todas direcciones, la teoría del éter explica estos mismos resultados introduciendo variaciones en la velocidad de la luz en direcciones diferentes, y además, un cambio compensatorio en los instrumentos del laboratorio, dependiendo cada variación de la velocidad del laboratorio a través del éter. Los efectos de ambos cambios se cancelan mutuamente dando un resultado nulo. Es obvio que la explicación dada por la teoría de éter de los resultados observados es innecesariamente complicada. Para dar cuenta de los resultados observados se necesita especificar un parámetro, la velocidad del laboratorio respecto al éter. Sin embargo, sea cual sea el valor que demos a dicha velocidad referida al éter obtenemos siempre las mismas predicciones experimentales, porque los efectos de la velocidad se cancelan mutuamente. De este modo, no existe ningún experimento que podamos realizar para medir la velocidad del laboratorio respecto al éter. La teoría de Einstein que niega sin más la existencia de semejante sistema tan huidizo resulta aparentemente más simple como hipótesis explicativa, y por tanto suele ser la preferida.

En situación parecida está el caso de la relatividad general. Con ella se explican las trayectorias curvas de luz y de las partículas afirmando que siguen las geodésicas nulas y cronomorfas de un espacio-tiempo curvo. Los postulados alternativos de un espacio-tiempo plano más fuerzas interpreta estas trayectorias curvas como el resultado de fuerzas que desvían a las partículas de las verdaderas geodésicas rectilíneas del espacio-tiempo plano real. Si se examinan detenidamente ambas teorías se ve que por cada universo posible descrito por un espacio-tiempo curvo corresponden una infinidad de universos describibles en términos de un espacio-tiempo plano más campos de fuerzas, del mismo modo en que anteriormente existían infinitos mundos con sistemas de éter más compensación por cada único mundo con espacio-tiempo minkowskiano relativista restringido. Siendo una vez más, como en el caso anterior, totalmente indistinguibles los infinitos universos con espacios-tiempos planos más campos. Esto no es más que una generalización de algo que Maxwell ya observó con relación a la teoría prerrelativista de la gravitación. Supongamos que existe en el universo un campo gravitatorio que fuese constante en todo lugar. Todo el universo material estaría, entonces, moviéndose con aceleración constante en este mundo. Aunque todos los objetos estarían siendo acelerados, esta aceleración, al contrario que la acele- 
ración normal, no podría ser ni detectada, ni medida, debido a que todos los aparatos de medida sufrirían la misma aceleración que el universo mismo. La existencia de un campo gravitatorio constante aún en caso de existir es un hecho indetectable.

Pero en la relatividad general no se postula la existencia de ningún campo gravitatorio y los infinitos mundos observacionalmente indistinguibles de la teoría anterior pasan a confundirse con un único mundo con espacio-tiempo curvo. En un sentido importante, las teorías einstenianas estándar son más simples que sus alternativas observacionalmente indistinguibles.

En principio se deberían dar por válidas todas estas teorías que son observacionalmente indistinguibles, pero si las dotamos previamente de alguna plausibilidad intrínseca que las distinga nos veremos abocados a decantarnos preferentemente por unas teorías en detrimento de otras.

No deja de ser legítima la postura de quienes se oponen a dar por válida esta solución al problema de Poincaré. Al fin y al cabo, ¿qué nos induce a pensar que la teoría más simple, aun cuando la noción de simplicidad pueda resultar coherente, es la que se debería adoptar como verdaderamente descriptora del mundo? Por supuesto que se podría preferir la teoría más simple por su inquietante atractivo estético, pero sería un error por nuestra parte entender la complejidad como una señal inequívoca de falsedad equiparable a la incapacidad de una teoría de acomodarse a los datos experimentales. Parece razonable que quien adoptara la postura realista de que hay un mundo independiente de nuestras teorías no debería considerar la simplicidad de una teoría como pauta para darla por verdadera.

\section{Consideraciones positivistas}

Diametralmente opuesto a estos enfoques del problema de Poincaré está la alternativa de tratar de socavar el desafío escéptico negando que haya realmente teorías diferentes entre las que se deba elegir. Partiendo de la afirmación de que las consecuencias observacionales deben determinar la identidad de las teorías se llega a la conclusión de que todas las supuestas teorías alternativas son realmente una única teoría y que las aparentes diferencias se dan tan solo por la forma elegida de expresar las mismas cosas sobre el mundo. 
La idea aquí expuesta es clara. Se pueden imaginar casos en los que dos expresiones de la teoría entran en conflicto entre sí, de tal modo que aparentemente las dos no pueden ser correctas, pero tal conflicto puede ser solamente ficticio. Es el resultado de utilizar los mismos términos con valores semánticos diferentes en ambas expresiones de la teoría. Si se propusieran dos teorías en las que todos los términos fueran idénticos y en las que únicamente se intercambiaran las palabras, por ejemplo, anión y catión, nos daríamos cuenta de inmediato de que ambas teorías propuestas son la misma. Lo que se ha hecho, es repetir lo mismo pero con los significados de dos palabras claves intercambiados. Si, por otra parte, se admite que todo el contenido real de una teoría viene dado por sus consecuencias observacionales y que dos teorías cualesquiera con las mismas consecuencias observacionales equivalen a una misma teoría, se concluye, ahora necesariamente, que las diferencias aparentes entre las dos expresiones de la teoría se deben únicamente a meras diferencias en los significados de algunos de los términos involucrados. Esto que se acaba de decir tiene cabida entre la enorme variedad de explicaciones positivas de lo que es una teoría y el significado teórico. Se puede sugerir que los términos en que se expresa una teoría, para que tengan un significado auténtico, deben recibir una definición individual acuñado por completo dentro del vocabulario que hace referencia a aquello que es observable. Así, si se tienen dos teorías aparentemente diferentes, o lógicamente incompatibles, pero que tienen las mismas consecuencias observacionales se podría averiguar qué términos de ambas formulaciones difieren en sus significados. En el caso de la relatividad podría decirse que el hecho de que los rayos de luz recorran geodésicas espacio-temporales nulas equivale a afirmar que la definición de geodésica espacio-temporal es la trayectoria de un rayo de luz. En la teoría alternativa de espacio-tiempo plano más fuerzas los rayos de luz no recorren geodésicas nulas, luego el término geodésica nula debe significar algo diferente en esta formulación, que no tiene nada que ver con el significado otorgado en la otra teoría. Un problema añadido a esta forma de entender el problema es el de si es posible que cualquier proposición de una teoría pueda ser traducida a otra en términos de otra teoría observacionalmente indistinguible. Mejor aún, que cualquier proposición de una teoría pueda ser traducida a una expresada únicamente en términos observacionales. En caso de que esta demanda de estricta traducibilidad proposición por proposición sea demasiado severa, siempre quedaría el consuelo de pensar que en una teoría lo que realmente tiene significado es la propia teoría como un todo y que este significado se agota completamente con sus consecuencias observacionales. Arguyendo de este modo, se concluye que en el caso 
de las teorías gravitacionales de espacio-tiempo curvo y plano carece de sentido preguntarse por los términos que han cambiado de significado de una teoría a otra. Como las dos teorías tienen las mismas consecuencias observacionales, consideradas como un todo global dicen las mismas cosas.

Esta forma de pensar rebosa, de una manera ilusoria, todo lo observable. Cuando se da a una teoría preferencia frente a otra observacionalmente equivalente, lo que realmente se está haciendo es elegir la manera que más nos gusta de expresar una teoría. Digamos que es como elegir un sistema de coordenadas para localizar los sucesos en lugar de otro. En contra de lo que pensaría un realista, para quien la simplicidad es un indicador intrínseco de credibilidad de una teoría, un positivista entiende la diferencia de simplicidad como simplicidad en la forma de expresión. No se trata de una diferencia de simplicidad como la que se pueda dar entre una ecuación de primer grado y una de segundo. Según esto, las teorías no son más que compendios lingüísticos, aunque no creo que fuera esto lo que Poincaré tenía en mente cuando planteó su convencionalismo ante la elección de la geometría que describe el mundo.

Llevando este enfoque a sus últimas consecuencias nos aparece un problema de difícil solución. Cuando nos fijamos en la parte inobservable de las teorías observacionalmente equivalentes, nos podemos dar cuenta que dicen cosas bastante incompatibles acerca de la estructura de lo inobservable. Entonces, si la equivalencia observacional nos ha bastado para postular la equivalencia entre ambas teorías, deberíamos vernos obligados a considerar aquello que dicen sobre lo inobservable de un modo meramente referencial. Si una teoría que afirma que el espacio-tiempo es curvo es equivalente a otra que afirma que el espacio-tiempo es plano, entonces las dos teorías están utilizando el espacio-tiempo como un objeto instrumental para poder generar un orden legal entre los observables que predicen a los que somos sensibles. Se debería así, considerar al espacio-tiempo como un objeto al que no se le puede atribuir ninguna característica. Se puede ver esto considerando el hecho de que si dos expresiones cualesquiera de teorías con un mismo contenido observacional son enteramente equivalentes, entonces el simple conjunto de consecuencias observacionales comunes a ambas teorías es equivalente a las dos.

Aparentemente, el considerar suficiente la equivalencia observacional para dar por válida la equivalencia total parece llevarnos a concluir un irrealismo radi- 
cal sobre lo inobservable. Las referencias a todo aquello que no es directamente accesible a la inspección observacional se convierte en una referencia ficticia, y las descripciones de las estructuras de lo inobservable se tornan en meras maneras de hablar y no en descripciones de algo realmente constitutivo del universo. Fuera del ámbito de la geometría esta conclusión nos conduciría a cuestionarnos la existencia de electrones y cuarks entre otras cosas. Más aún, considerar como directamente observable tan sólo aquello que consiste en datos sensoriales nos puede llevar a la concepción de que todo el mundo físico es una ficción. Sin duda, un irrealismo tan radical sobre la existencia del mundo es un precio muy alto a fin de evitar el desafío a nuestro conocimiento de la estructura geométrica de nuestro mundo.

\section{Consideraciones pragmáticas}

Las opciones que han sido expuestas no agotan todas las posibles consideraciones que se pueden plantear ante la cuestión de Poincaré. Se puede argumentar en favor de la idea de que los debates entre realistas e irrealistas descansan todos en confusiones. Por una parte, se puede regresar al escepticismo sobre la posibilidad de hacer de las consecuencias observables una clase preferente de las consecuencias de una teoría. Por otra parte, se puede afirmar que preocuparse por cuál de las posibles teorías alternativas es la verdadera tal vez sea una cuestión carente de sentido. Se sabe que existen varias descripciones que merecen, en función de algún conjunto de datos, ser razonables de creer. Supongamos que consideramos estas descripciones como verdaderamente incompatibles, no sólo por asumir que son meras variantes lingüísticas, como los positivistas nos harían creer. Si se eligiera una de tales teorías se declararían verdaderas sus consecuencias y falsas las consecuencias de sus rivales incompatibles. Si se hubiera elegido alguna de las otras alternativas, se tendrían que haber cambiado nuestras valoraciones acerca de que aserciones eran verdaderas y cuales falsas, manteniéndonos igualmente razonables. La pregunta que se nos viene ahora la cabeza es ¿qué es de todos modos la verdad? Tal vez Poincaré tuviera razón al afirmar que la geometría del mundo era una cuestión de convención en el sentido de que nos toca a nosotros elegir una de las opciones razonables a nuestra disposición y sobre la base de esto declarar verdaderas sus consecuencias. 


\section{Bibliografía}

Ben-Menahem, Y. 2001, Brit. J. Phil. Sci., 52, pp. 471-513.

Friedman, M. 1991, Fundamentos de las Teortas del Espacio-Tiempo. Madrid. Alianza Editorial.

Havas, P. 1964, Rev. Mod. Phys., 36, pp. 938-965.

PoINCARE, H. 2002, Ciencia e Hipótesis. Madrid. Espasa Calpe.

SKLAR, L. 1994, Filosofia de la Física. Madrid. Alianza Editorial. 